\title{
A 3-D Phase Evolution Panorama Uncovered Using a Grid-in-a-Coin Cell Method for Conversion Reaction Electrodes in Lithium-ion Batteries
}

\author{
Huolin L. Xin ${ }^{1}$, and Feng Lin $^{2}$ \\ ${ }^{1}$ Center for Functional Nanomaterials, Brookhaven National Laboratory, Upton, NY 11973, USA \\ 2. Environmental Energy Technologies Division, Lawrence Berkeley National Laboratory, Berkeley, CA \\ 94720, USA
}

In recent years, in situ transmission electron microscopy (TEM) studies have been performed to investigate the electrochemically driven phase conversions in lithium-ion batteries. However, most of these studies primarily focused on non-volatile environments and lacked information on interfaces between commercially used organic liquid electrolytes and battery electrodes. Furthermore, due to limitations of TEM sample geometry in these studies, electrode-electrolyte interfaces were simplified as a point/line contact and thus failed to represent the electrode materials' three-dimensional (3-D) immersion in electrolyte that occurs in practical battery cells. Other groups have attempted quasi in situ observation of energy materials supported on TEM grids in liquid electrochemical cells. However, due to the limited loading allowed on a TEM grid, an accurate and reproducible charging/discharging rate (C-rate) control is difficult to achieve but is essential for lithium-ion battery systems. Here, we report a direct visualization of phase conversion in a high performance anode material in a coin cell configuration [1]. In the coin cell, we pressed a holey carbon supported TEM grid, which was loaded with a small amount of the anode material, against the bulk anode to ensure the materials on the grid are submerged in the same electrolyte environment as the bulk anode (Fig. 1). This configuration allows us to accurately control the C-rate for cycling; additionally the TEM grid can be dissembled from the cell to facilitate TEM imaging and spectroscopy. This grid-in-a-coin cell methodology has enabled direct probing of the 3-D morphology and charge state distribution of the anode material and evolution of AEI at different stages of electrochemical cycling with atomic-scale TEM imaging, electron tomography, electron energy loss spectroscopic mapping as well as ensemble-averaged synchrotron X-ray spectroscopy.

Using this method, we uncovered a phase evolution panorama at multiple states of charge for nickel oxide nanosheets in lithium-ion batteries. We reconstruct the three-dimensional reaction fronts and find that, in a fully electrolyte immersion environment, phase conversion can nucleate from spatially distant locations on the same slab of material (Fig. 2 and 3). This finding dispels the current prevailing theory of homogeneous reaction in electrolyte-submerged samples. Moreover, we tracked the evolution of the surface chemistry, demonstrating that the SEI layer on the $\mathrm{NiO}$ anode has a reversible characteristic, which has not been directly visualized before (Fig. 4). This work uncovers the dominance of heterogeneous nucleation/reactions and their underlying atomic-scale kinetic mechanisms in realistic battery phase conversion reactions. It has implications for resolving the origin of inhomogeneous charge distribution in large-format battery electrodes. [1][2].

\section{References}

[1] F. Lin et al, and H. L. Xin, Nature Communications, DOI: 10.1038/ncomms4358 (2014)

[2] Research carried out in part at the Center for Functional Nanomaterials, Brookhaven National

Laboratory, which is supported by the U.S. Department of Energy (DOE), Office of Basic Energy 
Sciences, under Contract No. DE-AC02-98CH10886. This study also made use of NCEM facilities supported by the DOE under Contract No. DE-AC02-05CH11231, and Stanford Synchrotron Radiation Lightsource, a Directorate of SLAC National Accelerator Laboratory and an Office of Science User Facility operated for the U.S. Department of Energy Office of Science by Stanford University.

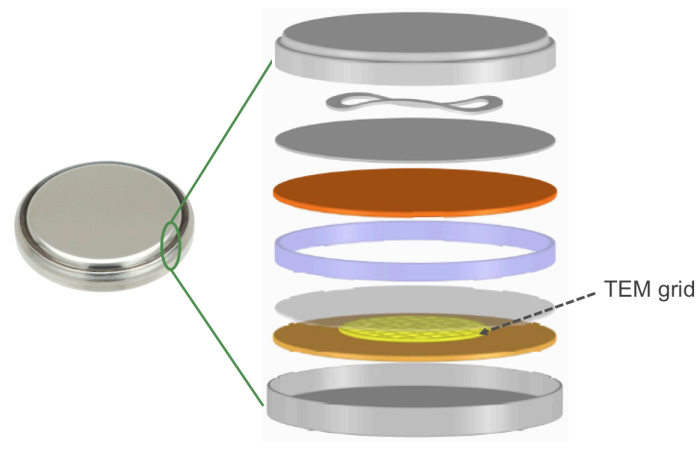

Figure 1. Schematic illustration of a coin cell design loaded with a TEM grid
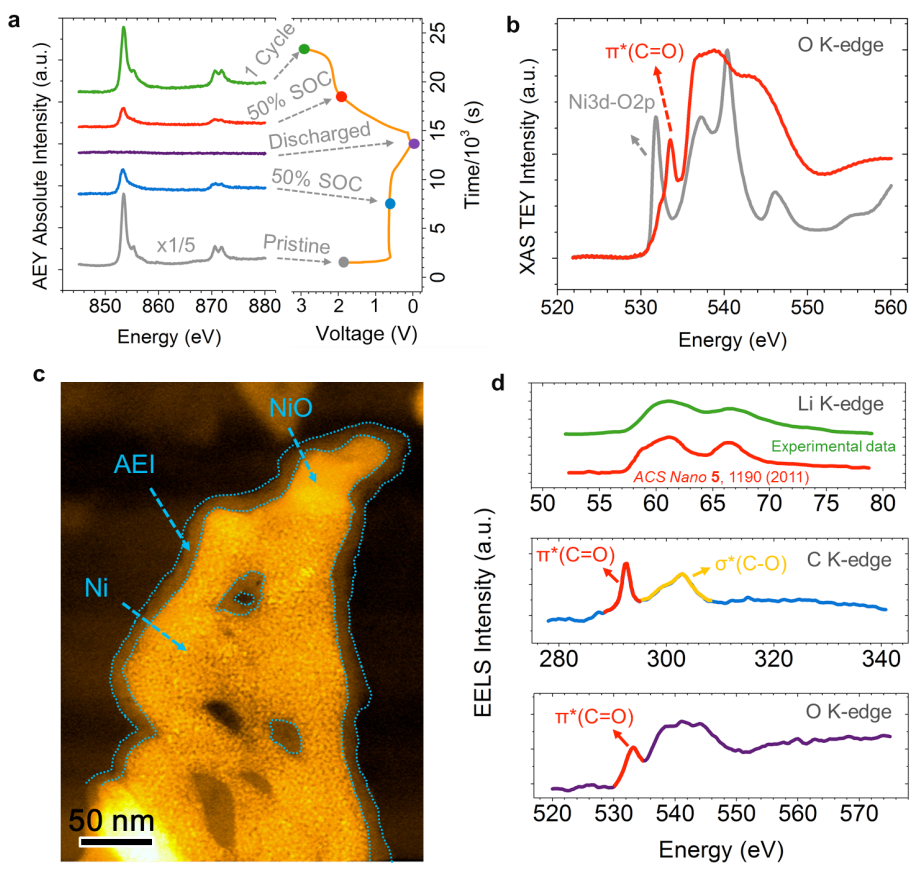

Figure 4. Chemical evolution at the surface of $\mathrm{NiO}$ nanosheets. (a) Auger-yield XAS spectra for electrodes at different states of charge. (b) Total-electron-yeild XAS $\mathrm{O}$ K-edge spectra for electrodes in the pristine state (gray) and in the $50 \%$ state of charge (red). (c) STEM image of a $\mathrm{NiO}$ nanosheet during a discharging process. (d) low-dose rate Li K-edge, $\mathrm{C}$ K-edge and $\mathrm{O}$ K-edge EELS spectra for the AEI layer.
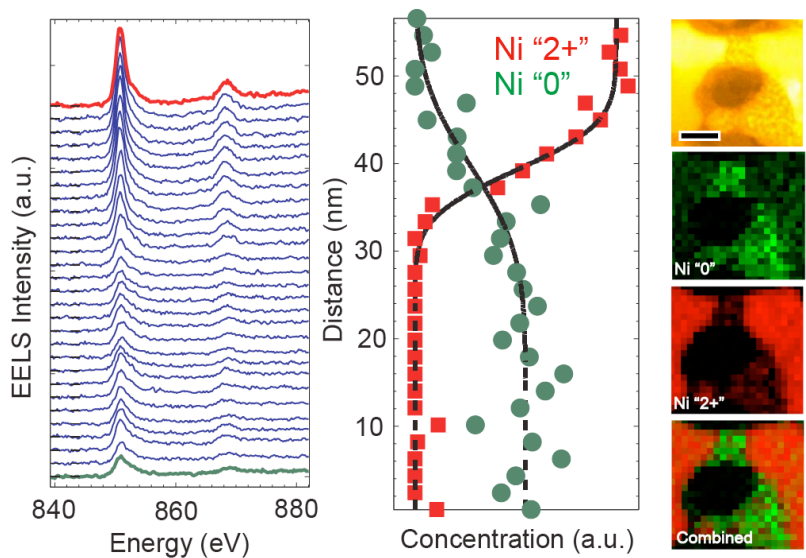

Figure 2. STEM-EELS spectroscopic imaging of the $\mathrm{NiO}$ at $50 \%$ state of charge.

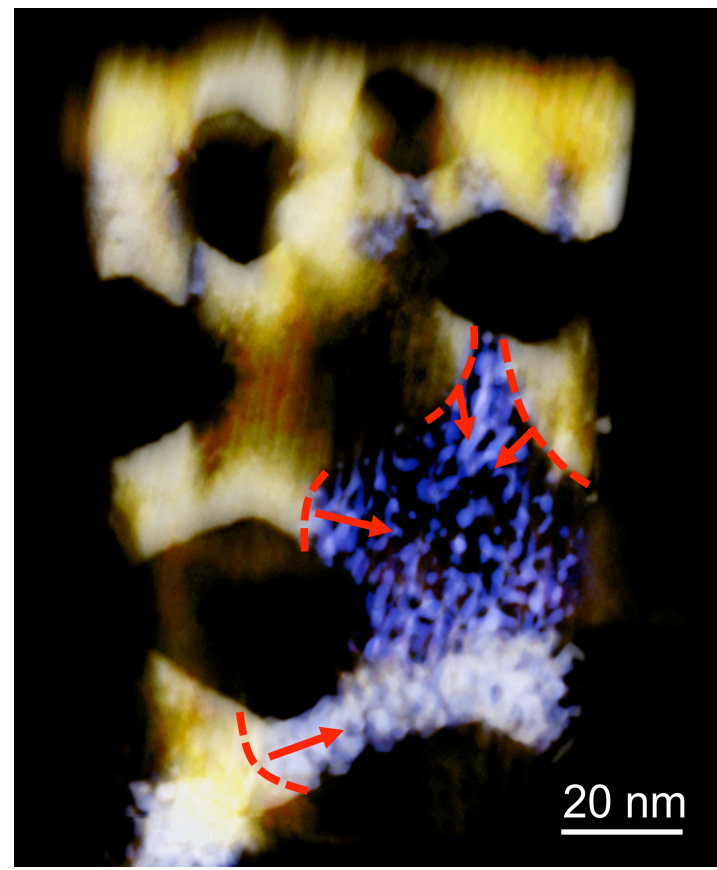

Figure 3. Tomographic reconstruction of a $\mathrm{NiO}$ sheet with a $50 \%$ state of charge. The contours and arrows in the Feature Image highlight the phase conversion fronts that had simultaneously propagated in a single $\mathrm{NiO}$ nanosheet. 